TRANSACTIONS OF THE

AMERICAN MATHEMATICAL SOCIETY

Volume 348, Number 6, June 1996

\title{
REAL CONNECTIVE K-THEORY AND THE QUATERNION GROUP
}

\author{
DILIP BAYEN AND ROBERT R. BRUNER
}

\begin{abstract}
Let $k o$ be the real connective K-theory spectrum. We compute $k o_{*} B G$ and $k o^{*} B G$ for groups $G$ whose Sylow 2-subgroup is quaternion of order 8. Using this we compute the coefficients $t(k o)_{*}^{G}$ of the $G$ fixed points of the Tate spectrum $t(k o)$ for $G=S l_{2}(3)$ and $G=Q_{8}$. The results provide a counterexample to the optimistic conjecture of Greenlees and May [9, Conj. 13.4], by showing, in particular, that $t(k o)^{G}$ is not a wedge of Eilenberg-Mac Lane spectra, as occurs for groups of prime order.
\end{abstract}

\section{INTRODUCTION}

Let $G$ be a group whose Sylow 2-subgroup is quaternion of order 8. The real connective K-homology of the classifying space $B G$ is of interest because a number of geometric invariants of manifolds with fundamental group $G$ lie in $k o_{*} B G$. In particular, Stolz shows in [14] that for fundamental groups which are finite 2groups the question of positive scalar curvature reduces to $k o_{*}(B G)$. Using this, the Gromov-Lawson conjecture has been settled for manifolds whose fundamental group has periodic cohomology $[5,6,7,15]$.

Further, the periodic K-theory of $B G$ is determined by the real, complex and quaternionic representations of $G$. The connective K-theory provides more refined invariants of $G$ whose algebraic meaning would be interesting to discern.

The approach we use applies quite generally to the computation of the kohomology and cohomology of $B G$, and is as follows. We localize at 2 throughout, let $H^{*}$ be $H^{*}(; \mathbf{Z} / 2)$, and let $\mathcal{A}$ and $\mathcal{A}(1)$ be the mod 2 Steenrod algebra and its subalgebra generated by $S q^{1}$ and $S q^{2}$.

1. Compute the periodic K-theory $K O_{*} B G$ in terms of the representation ring of $G$, using the result of Stolz and Rosenberg quoted as Theorem 2 below.

2. Decompose $B G$ stably using the results of Martino and Priddy quoted as Theorem 1.

3. Describe the corresponding summands of $H^{*} B G$ as $\mathcal{A}(1)$-modules.

4. Compute the $E_{2}$-term of the Adams spectral sequence

$$
\operatorname{Ext}_{\mathcal{A}}\left(H^{*}(k o \wedge X), \mathbf{Z} / 2\right) \cong \operatorname{Ext}_{\mathcal{A}(1)}\left(H^{*} X, \mathbf{Z} / 2\right) \Longrightarrow k o_{*} X
$$

for each summand $X$ of $B G$.

Received by the editors August 10, 1994.

1991 Mathematics Subject Classification. Primary 19L41, 19L47, 19L64, 55N15, 55R35, 55Q91, 55M05.

Key words and phrases. Quaternion group, classifying space, connective K-theory, Tate cohomology.

(C)1996 American Mathematical Society 
5. Determine the differentials and extensions in the Adams spectral sequence of each summand using the Bockstein spectral sequence of $k o \wedge X$ and the fact that, after inverting the Bott element, $k o_{*}$ becomes $K O_{*}$, which is known by steps 1 and 2 .

6. Write $X$ as a convenient direct limit, $X=\lim X_{n}$, compute each $k o^{*} X_{n}$ using S-duality, and compute $k o^{*} X=\lim _{\leftarrow} k o^{*} X_{n}$.

In the last section we provide some background information on Tate theories and compute $t(k o)_{*}^{G}$ using the norm sequence

$$
\cdots \rightarrow k o_{n} B G_{+} \rightarrow k o^{-n} B G_{+} \rightarrow t(k o)_{n}^{G} \rightarrow k o_{n-1} B G_{+} \rightarrow \cdots .
$$

The authors wish to thank Stephan Stolz for numerous helpful conversations. and for interesting us in these matters, and Peter Gilkey for sharing his calculations of the eta invariant at a crucial moment.

Some of the results here are a part of the first author's thesis [3].

\section{Decomposition of $B G$ and group representations of $Q_{8}$}

Theorem 1 (J. Martino and S. Priddy [10]). Let G be a finite group with Sylow 2subgroup the quaternion group $Q_{8}$. Then the complete 2-local stable decomposition of $B G$ into indecomposable summands is either

1. $B G \simeq B S l_{2}(3)$, or

2. $B G \simeq B S l_{2}(3) \vee \Sigma^{-1} B S^{3} / B N \vee \Sigma^{-1} B S^{3} / B N$, where $N$ is the normalizer of a maximal torus in $S^{3}$.

Note that $B Q_{8}$ itself is the larger of these. Since $Q_{8}$ acts freely on the 3 -sphere, its cohomology is periodic with period 4 . Explicitly,

$$
\begin{aligned}
H^{*}\left(B Q_{8}\right)= & \mathbf{Z} / 2[x, y, w] /\left(x^{2}+x y+y^{2}, x^{2} y+x y^{2}\right), \\
& |x|=|y|=1, \text { and }|w|=4, \text { and } \\
H^{*}\left(B S l_{2}(3)\right)= & \mathbf{Z} / 2[w] \otimes \wedge(\tilde{x}),|w|=4,|\tilde{x}|=3 .
\end{aligned}
$$

The inclusion of the Sylow 2-subgroup $Q_{8} \rightarrow S l_{2}(3)$ induces an isomorphism in cohomology in dimensions 3 and $4 \bmod 4$, with $\tilde{x}$ mapping to $x^{2} y=x y^{2}$. The two copies of $\Sigma^{-1} B S^{3} / B N$ are embedded as

$$
\left\{x^{\epsilon} w^{k} \mid \epsilon=1,2 \text { and } k \geq 0\right\}
$$

and

$$
\left\{y^{\epsilon} w^{k} \mid \epsilon=1,2 \text { and } k \geq 0\right\} .
$$

The Bocksteins are given by $\beta_{1} x=x^{2}, \beta_{1} y=y^{2}$, and $\beta_{3} \tilde{x}=w$ by [13]. Thus, $\Sigma^{-1} B S^{3} / B N$ is composed of $\bmod 2$ Moore spaces and $B S l_{2}(3)$ is composed of mod 8 Moore spaces.

The real representations of $Q_{8}$ determine the periodic real K-theory of $B Q_{8}$ by the following result.

Theorem 2 (S. Stolz and J. Rosenberg [15]). Let $G$ be a finite p-group. Then $K O_{*}(B G)$ splits as a $K O_{*}$-module into the direct sum of copies of

$$
0 \quad 1
$$

$$
0 \quad(\mathbf{Z} / 2)
$$$$
2
$$

$(\mathbf{Z} / 2)$

$0 \quad \mathbf{Z} / p^{\infty}$

0
0

$\begin{array}{cc}3 & 4 \\ \mathbf{Z} / p^{\infty} & 0 \\ \mathbf{Z} / p^{\infty} & 0\end{array}$

5
0
$\mathbf{Z} / p^{\infty}$
$(\mathbf{Z} / 2)$

6

0

7
$\mathbf{Z} / p^{\infty}$
$\mathbf{Z} / p^{\infty}$

$(\mathbf{Z} / 2) \quad \mathbf{Z} / p^{\infty}$

$(\bmod 8)$ 
one for each nontrivial irreducible real, complex, or quaternionic representation, respectively. Here $(\mathbf{Z} / 2)$ is $\mathbf{Z} / 2$ if $p=2$ and is 0 otherwise.

Note that, when $p=2$, these three $K O_{*}$-modules are $K O_{*}\left(\mathbf{R} \mathbf{P}^{\infty}\right), K U_{*}\left(\mathbf{R P}^{\infty}\right)$, and $K S p_{*}\left(\mathbf{R P}^{\infty}\right)$.

$Q_{8}$ has three nontrivial 1-dimensional representations and one irreducible 2dimensional representation. By the Frobenius-Schur indicator, the 1-dimensional representations are all real, and the 2-dimensional representation is quaternionic. (Equivalently, we could observe that the Wedderburn decomposition of the real group ring $\mathbb{R}\left[Q_{8}\right]$, is $\mathbb{R}^{4} \oplus \mathbb{H}$.) Comparing the splittings of $K O_{*} B Q_{8}$ given by the preceding two theorems, the following corollary is immediate.

Corollary 3. As $K O_{*}$-modules,

$$
\begin{aligned}
K O_{*}\left(\Sigma^{-1} B S^{3} / B N\right) & \cong K O_{*}\left(\mathbf{R P}^{\infty}\right) \\
K O_{*}\left(B S l_{2}(3)\right) & \cong K O_{*}\left(\mathbf{R P}^{\infty}\right) \oplus K S p_{*}\left(\mathbf{R P}^{\infty}\right), \\
K O_{*}\left(B Q_{8}\right) & \cong \bigoplus_{3} K O_{*}\left(\mathbf{R P}^{\infty}\right) \cdot \oplus K S p_{*}\left(\mathbf{R P}^{\infty}\right)
\end{aligned}
$$

\section{The Bockstein differentials in the Adams spectral sequence}

Recall that $H^{*} k o=\mathcal{A} \otimes_{\mathcal{A}(1)} \mathbf{Z} / 2$ [16]. As a result, the Kunneth isomorphism

$$
H^{*}(k o \wedge X) \cong \mathcal{A} \otimes_{\mathcal{A}(1)} \mathbf{Z} / 2 \otimes H^{*} X \cong \mathcal{A} \otimes_{\mathcal{A}(1)} H^{*} X
$$

and the adjunction

$$
\operatorname{Hom}_{\mathcal{A}}\left(\mathcal{A} \otimes_{\mathcal{A}(1)} M, N\right) \cong \operatorname{Hom}_{\mathcal{A}(1)}(M, N)
$$

give the ordinary mod 2 Adams spectral sequence the form

$$
\operatorname{Ext}_{\mathcal{A}(1)}^{s, t}\left(H^{*} X, \mathbf{Z} / 2\right) \Longrightarrow k o_{t-s} X .
$$

Recall also that for $X=S^{0}$ the spectral sequence collapses at $E_{2}$ to give

$$
\begin{aligned}
E_{2}=E_{\infty}= & \mathbf{Z} / 2\left[h_{0}, h_{1}, w, b\right] /\left(h_{0} h_{1}, h_{1}^{3}, h_{1} w, w^{2}+h_{0}^{2} b\right), \\
& \left|h_{0}\right|=(0,1),\left|h_{1}\right|=(1,1),|w|=(4,3),|b|=(8,4),
\end{aligned}
$$

where $|x|=(t-s, s)$ specifies the geometric degree $t-s$ and the Adams filtration $s$. We will refer, ambiguously, to $b$ and to a generator $\beta \in k o_{8}\left(S^{0}\right)$ detected by $b$, as 'the Bott element', since multiplication by its image in $K O_{8}\left(S^{0}\right)$ is the Bott periodicity isomorphism.

As an $\mathcal{A}(1)$-module, $H^{*}\left(\Sigma^{-1} B S^{3} / B N\right)$ is a direct sum,

$$
H^{*}\left(\Sigma^{-1} B S^{3} / B N\right)=\bigoplus_{n \geq 1} H^{*} M^{4 n-2}(2),
$$

where $M^{n}(k)=S^{n-1} \cup_{k} e^{n}$ is the $\bmod k$ Moore space with top cell in dimension $n$. It is well known, and easy to calculate, that, as an $E_{2}\left(S^{0}\right)$-module,

$$
\begin{aligned}
E_{2}\left(M^{1}(2)\right) & =E_{\infty}\left(M^{1}(2)\right) \\
& =\left\langle x, y \mid h_{0} x=0, h_{1}^{2} x=h_{0} y, h_{1}^{2} y=w x, w y=0\right\rangle \\
& \Longrightarrow k o_{*}\left(M^{1}(2)\right),
\end{aligned}
$$

with $|x|=(t-s, s)=(0,0)$ and $|y|=(2,1)$. It follows that $E_{2}\left(\Sigma^{-1} B S^{3} / B N\right)$ is the direct sum of copies of $E_{2}\left(M^{4 n-2}(2)\right)$ generated by $x_{n}$ and $y_{n}$ for $n \geq 1$ with $\left|x_{n}\right|=(4 n-3,0)$ and $\left|y_{n}\right|=(4 n-1,1)$. (See Figure 1.) 


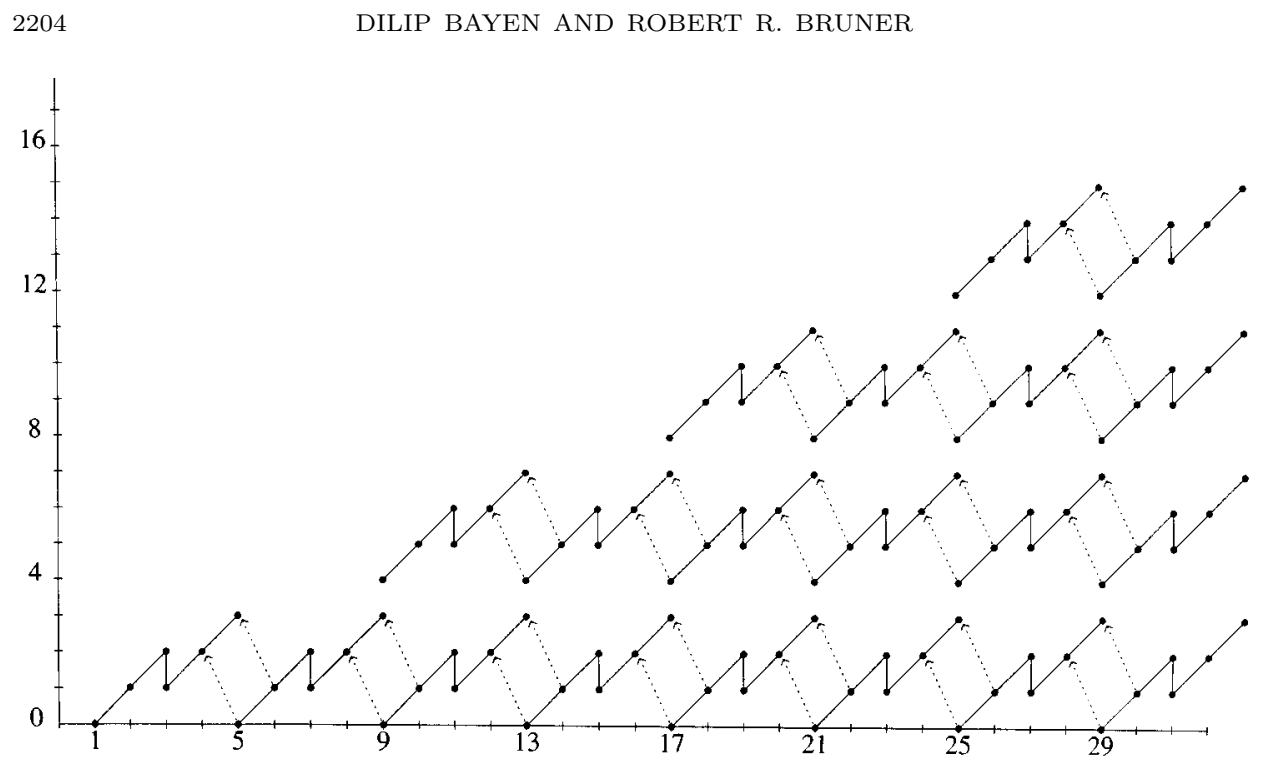

Figure 1. $E_{2}$ for $k o_{*} \Sigma^{-1} B S^{3} / B N$

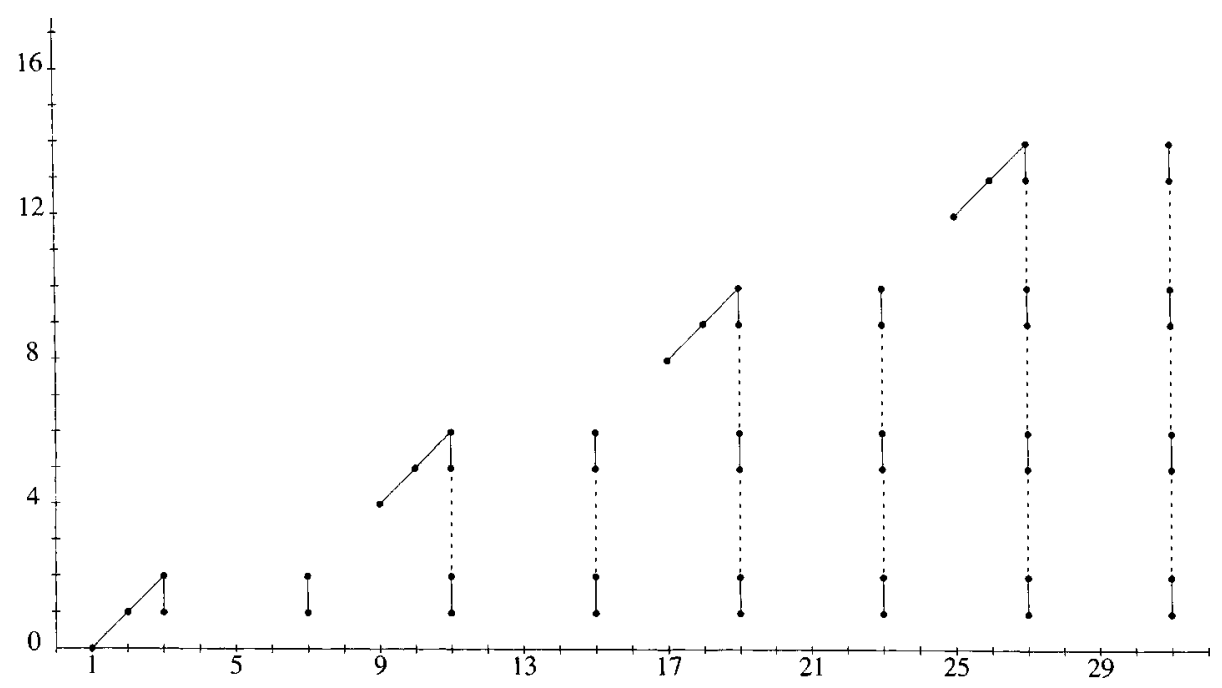

FiguRE 2. $E_{3}=E_{\infty}=E^{0}\left(k o_{*} \Sigma^{-1} B S^{3} / B N\right)$

As an $\mathcal{A}(1)$-module, $H^{*}\left(B S l_{2}(3)\right)$ is a direct sum of $\mathbf{Z} / 2$ 's, one in each dimension congruent to 3 or $4 \bmod 4$. Thus, $E_{2}\left(B S l_{2}(3)\right)$ is the corresponding sum of copies of $E_{2}\left(S^{0}\right)$, one starting in each dimension congruent to 3 or $4 \bmod 4$. The theorem of [11] tells us that, for $r \geq 2$, there is a 1-1 correspondence between infinite $h_{0^{-}}$ towers in $E_{r}$ of the Adams spectral sequence, i.e. sets $\left\{h_{0}^{i} x \mid i \geq 0\right\}$ with $h_{0}^{i} x \neq 0$ for all $i$, and the $E_{r}$-term of the homology Bockstein spectral sequence, and that, under this correspondence, the Adams $d_{r}$ connecting these towers corresponds to the Bockstein $\beta_{r}$. We will compute the cohomology Bockstein spectral sequence and dualize. For ko, the Bockstein spectral sequence is well known ([12]) to collapse 
at $E_{2}$, with $E_{2}=E_{\infty}$ spanned by the equivalence classes of $S q^{4 n} \iota$, where $\iota \in$ $H^{0} k o$ is the generator. The Bockstein spectral sequence of $B S l_{2}(3)$ has $E_{1}=E_{3}$, $\beta_{3}\left(\tilde{x} w^{k}\right)=w^{k+1}$, and $E_{4}=E_{\infty}=0$. Tensoring these, we find that the Bockstein spectral sequence for $k o \wedge B S l_{2}(3)$ has

$$
E_{2}=E_{3}=\left\{S q^{4 n} \iota \mid n \geq 0\right\} \otimes \wedge(\tilde{x}) \otimes \mathbf{Z} / 2[w]
$$

with

$$
\beta_{3}\left(S q^{4 n} \iota \otimes \tilde{x} w^{k}\right)=S q^{4 n} \iota \otimes w^{k+1}
$$

and $E_{4}=E_{\infty}=0$. Thus, the towers in the Adams spectral sequence will be annihilated by Adams $d_{3}$ 's. Examining $E_{3}$ we find that this $d_{3}$ must be

$$
d_{3}\left(x_{4 n}\right)=\alpha h_{0}^{3} x_{4 n-1}+\beta w x_{4 n-5}
$$

with $\alpha, \beta \in\{0,1\}$ and $x_{i} \in E_{3}^{0, i}$. Comparison with the Adams spectral sequence for $k o_{*} M^{4 n}(8)$ shows that $\alpha=1$. In order to determine $\beta$, it will help to examine the isomorphisms

$$
\begin{aligned}
\operatorname{Ext}_{\mathcal{A}}\left(H^{*}(k o \wedge X), \mathbf{Z} / 2\right) & \cong \operatorname{Ext}_{\mathcal{A}}\left(H^{*}(k o) \otimes H^{*}(X), \mathbf{Z} / 2\right) \\
& \cong \operatorname{Ext}_{\mathcal{A}(1)}\left(H^{*}(X), \mathbf{Z} / 2\right)
\end{aligned}
$$

more carefully. The first isomorphism is induced by the usual Kunneth isomorphism $H^{*}(k o \wedge X) \cong H^{*} k o \otimes H^{*} X$, in which the tensor product has the diagonal $\mathcal{A}$ action. The second isomorphism applies generally to extended modules, and requires that the tensor product be given the 'extended' $\mathcal{A}$ action in which $\mathcal{A}$ acts only on the left factor, $H^{*} k o \cong \mathcal{A} \otimes_{\mathcal{A}(1)} \mathbf{Z} / 2$. The two isomorphisms can thus be combined only because of a third isomorphism $\theta$ between the diagonal and extended actions. For convenience of notation, note that if we let $\widehat{H^{*} X}$ denote $H^{*} X$ with trivial $\mathcal{A}$ action, i.e., $\mathcal{A}$ action pulled back along the augmentation $\mathcal{A} \rightarrow \mathbf{Z} / 2$, then the diagonal action on $H^{*} k o \otimes \widehat{H^{*} X}$ is the extended action. The isomorphism

$$
\theta: H^{*} k o \otimes \widehat{H^{*} X} \rightarrow H^{*} k o \otimes H^{*} X
$$

is $\theta(a \iota \otimes x)=\sum a^{\prime} \iota \otimes a^{\prime \prime} x$, with inverse $\theta^{-1}(a \iota \otimes x)=\sum a^{\prime} \iota \otimes \chi\left(a^{\prime \prime}\right) x$, where $\psi(a)=\sum a^{\prime} \otimes a^{\prime \prime}$. These matters are discussed nicely in [1, §III.16] and [2, 3.1]. To apply the theorem of [11] to towers in $\operatorname{Ext}_{\mathcal{A}(1)}\left(H^{*}(X), \mathbf{Z} / 2\right)$, the convenient Bockstein is therefore $\widehat{\beta}_{3}=\theta^{-1} \beta_{3} \theta$. We find

$$
\begin{aligned}
\widehat{\beta}_{3}\left(\iota \otimes \tilde{x} w^{k}\right) & =\iota \otimes w^{k+1}, \text { and } \\
\widehat{\beta}_{3}\left(S q^{4} \iota \otimes \tilde{x} w^{k-1}\right) & =S q^{4} \iota \otimes w^{k}+\iota \otimes w^{k+1} .
\end{aligned}
$$

Dualizing, we see that

$$
d_{3}\left(x_{4 n}\right)=h_{0}^{3} x_{4 n-1}+w x_{4 n-5} .
$$

Thus, in $E_{4}$, we have generators $x_{4 n-1}$ and $y_{4 n+1}$ for $n \geq 1$ with $\left|x_{4 n-1}\right|=(4 n-1,0)$ and $\left|y_{4 n+1}\right|=(4 n+1,1)$, and with the relation $w x_{4 n-1}=h_{0}^{3} x_{4 n+3}$ (and hence $\left.h_{0}^{2} b x_{4 n-1}=h_{0}^{6} x_{4 n+7}\right)$ for every $n>0$. See Figure 3 , in which we have replaced the class $b^{i} x_{4 n-1}$ by the sum $b^{i} x_{4 n-1}+h_{0}^{4 i} x_{8 i+4 n-1}$ to simplify the diagram. 


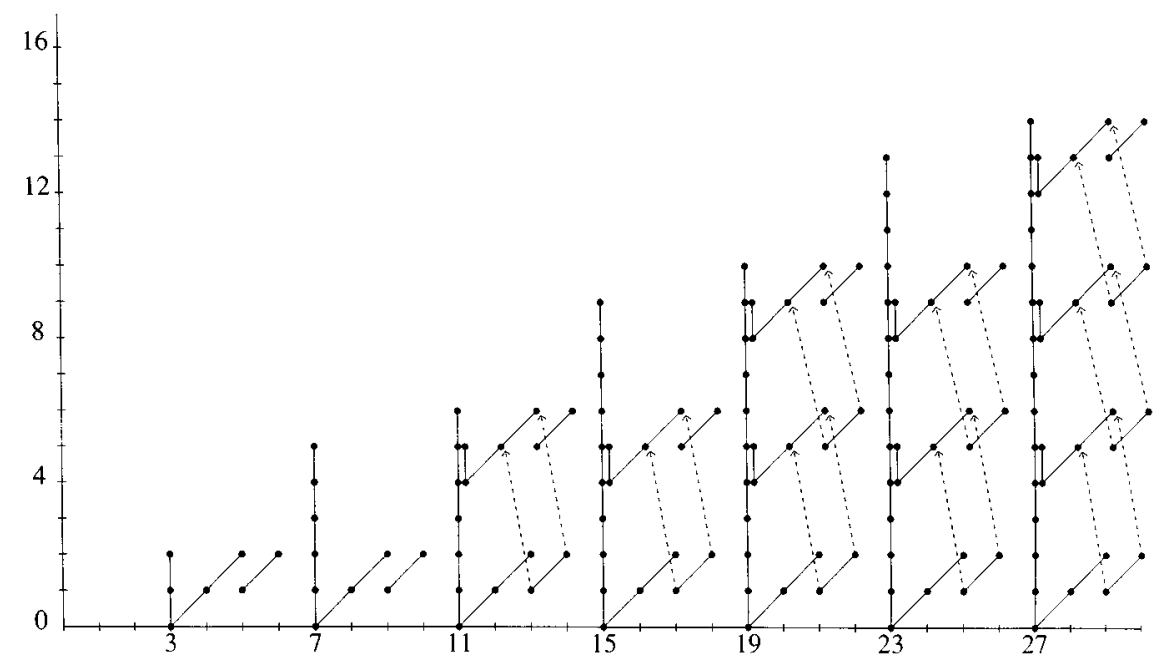

Figure $3 . E_{4}$ for $k o_{*} B S l_{2}(3)$

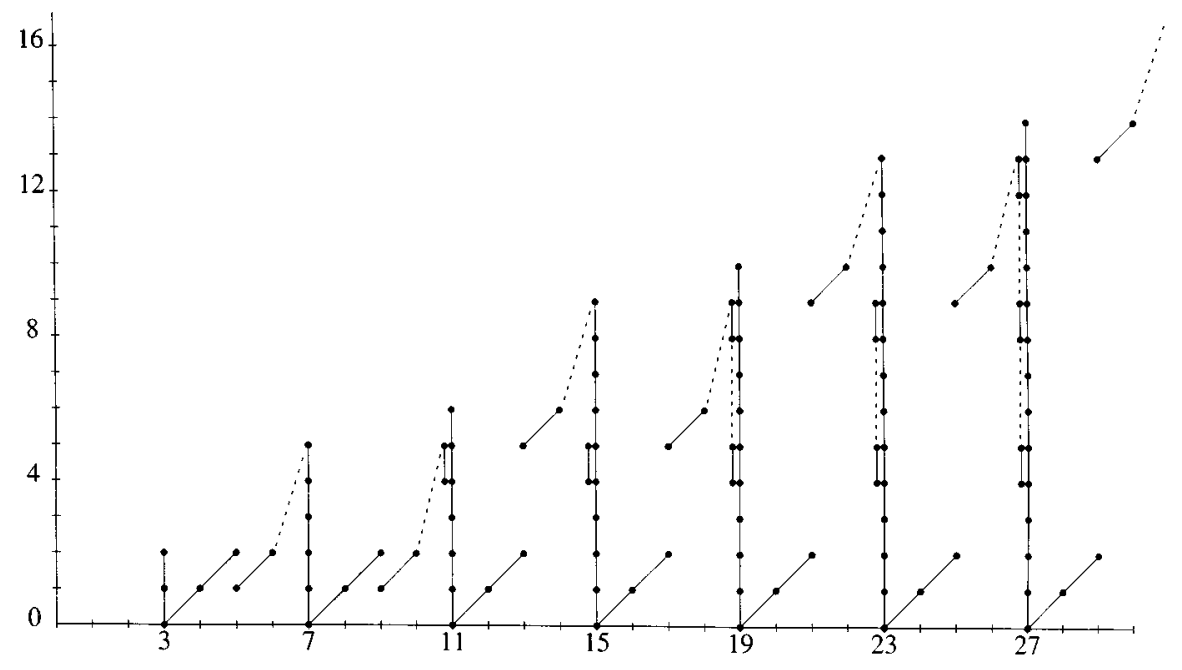

Figure 4. $E_{5}=E_{\infty}=E^{0}\left(k o_{*}\left(B S l_{2}(3)\right)\right)$

4. The $k o$-Homology of $\Sigma^{-1} B S^{3} / B N$

Theorem 4. In the Adams spectral sequence for $k_{*} \Sigma^{-1} B S^{3} / B N$,

$$
d_{2}\left(x_{n+1}\right)=h_{1} y_{n}
$$

and $E_{3}=E_{\infty}$. Furthermore, all possible extensions are nonzero. Therefore, for $n \geq 0$

$$
k o_{8 n+\epsilon} \Sigma^{-1} B S^{3} / B N=\left\{\begin{array}{lllr}
\mathbf{Z} / 2 & =\left\langle\beta^{n} x_{1}\right\rangle & \epsilon=1 \\
\mathbf{Z} / 2 & =\left\langle\eta \beta^{n} x_{1}\right\rangle & 2 \\
\mathbf{Z} / 2^{2 n+2} & =\left\langle y_{2 n+1}\right\rangle & 3 \\
\mathbf{Z} / 2^{2 n+2} & =\left\langle y_{2 n+2}\right\rangle & 7 \\
0 & & & 4,5,6,8
\end{array}\right.
$$


with $w y_{n}=4 y_{n+1}, \beta y_{n}=4 y_{n+2}$, and $\eta^{2} \beta^{n} x_{1}=2^{2 n+1} y_{2 n+1}$ for all $n>0$.

Proof. Recall that

$$
k o_{*}\left(\Sigma^{-1} B S^{3} / B N\right)\left[b^{-1}\right]=K O_{*} \Sigma^{-1} B S^{3} / B N \cong K O_{*} \mathbf{R P}^{\infty}
$$

and is therefore 0 in dimensions congruent to 4,5 , or $6, \bmod 8$. We will show that this forces $d_{2}\left(x_{2 n}\right)=h_{1} y_{2 n-1}$ for each $n>0$. Similarly, the fact that $K O_{*} \mathbf{R} \mathbf{P}^{\infty}$ has order 2 in dimensions 1 and $2 \bmod 8$, and is 0 in dimensions congruent to 0 $\bmod 8$ will imply that $d_{2}\left(x_{2 n+1}\right)=h_{1} y_{2 n}$.

We proceed inductively. First, consider $x_{2} \in E_{2}^{5,0}$. If $d_{2}\left(x_{2}\right) \neq h_{1} y_{1}$, then $b^{n} x_{2}$ survives to $E_{\infty}$ for each $n$. Since $K O_{5}=0, b^{n} x_{2}$ must be a boundary for large enough $n$. Choose such an $n$, and let $u_{i}=b^{n-i} x_{2 i+2}$ and $v_{i}=b^{n-i} h_{1} y_{2 i+1}$ in order to simplify the notation. In order for $u_{0}=b^{n} x_{2}$ to be a boundary, it must be $d_{r_{1}} h_{1} u_{t_{1}}=u_{0}$ for some $r_{1}$ and $t_{1}$. The fact that $u_{0}$ is not a multiple of $h_{1}$ implies that $h_{1} u_{t_{1}}$ must not be an $h_{1}$ multiple in $E_{r_{1}}$. Hence, $u_{t_{1}}$ must have supported an earlier nonzero differential $d_{r_{2}} u_{t_{1}}=v_{s_{2}}, r_{2}<r_{1}$, and we must have had $h_{1} v_{s_{2}}=0$ in $E_{r_{2}}$ to allow $h_{1} u_{t_{1}}$ to survive to $E_{r_{1}}$. This requires a still earlier differential $d_{r_{3}} h_{1} u_{t_{2}}=h_{1} v_{s_{2}}$ with $r_{3}<r_{2}<r_{1}$. To allow $v_{s_{2}}$ to survive to $E_{r_{2}}$, we find that $h_{1} u_{t_{2}}$ must not be an $h_{1}$ multiple in $E_{r_{3}}$. Continuing in this way, we find an infinite sequence of ever shorter differentials. This contradiction shows that $b^{n} x_{2}$ cannot be the target of a differential, and we conclude that $d_{2}\left(x_{2}\right)=h_{1} y_{1}$.

The remaining even differentials $d_{2}\left(x_{2 n}\right)=h_{1} y_{2 n-1}$ follow by induction on $n$, since the inductive hypothesis implies that this is the only nontrivial differential $x_{2 n}$ can support. The possibility that $x_{2 n}$ could be the target of a differential is ruled out exactly as for $x_{2}$.

The same argument also shows that $b^{n} x_{1}$ cannot be the target of any differential. Since $K O_{*} \Sigma^{-1} B S^{3} / B N \cong K O_{*} \mathbf{R P}^{\infty}$ as $K O_{*}$-modules, it follows that $b^{n} x_{1}$ and $b^{n} h_{1} x_{1}$ survive to nonzero classes in $E_{\infty}$. The remaining classes in stems congruent to 0,1 and 2 , mod 8 must vanish upon inverting the Bott element, and, hence, the same argument as for the even differentials establishes the odd differentials, $d_{2}\left(x_{2 n+1}\right)=h_{1} y_{2 n}$, for $n>0$.

This leaves no room for further differentials, so $E_{\infty}$ is as shown in Figure 2. The fact that each $K O_{i} \Sigma^{-1} B S^{3} / B N$ is cyclic, together with the fact that the Bott element acts monomorphically on $E_{\infty}$, implies that all possible extensions are nontrivial. Finally, the $k o_{*}$-module structure is evident from the Adams spectral sequence.

QED

\section{The ko-Homology of $\left.B S l_{2}(3)\right)$}

Theorem 5. In the Adams spectral sequence for $k o_{*}\left(B S l_{2}(3)\right)$

$$
d_{4}\left(y_{4 n+1}\right)=h_{1} b x_{4 n-9}
$$

and $E_{5}=E_{\infty}$. As a $k o_{*}$-module, $k o_{*}\left(B S l_{2}(3)\right)$ is generated by elements $y_{5}, y_{9}$, and $x_{4 n+3}$ for $n \geq 0$. Furthermore, for $n \geq 0$, 


$$
k o_{8 n+\epsilon} B S l_{2}(3)=\left\{\begin{array}{llr}
\mathbf{Z} / 2^{4 n+3} \oplus \mathbf{Z} / 2^{2 n} & =\left\langle x_{8 n+3}\right\rangle \oplus\left\langle\bar{x}_{8 n+3}\right\rangle & \epsilon=3 \\
\mathbf{Z} / 2 & =\left\langle\eta x_{8 n+3}\right\rangle & 4 \\
\mathbf{Z} / 2 \oplus \mathbf{Z} / 2 & =\left\langle\eta^{2} x_{8 n+3}\right\rangle \oplus\left\langle\beta^{n} y_{5}\right\rangle & 5 \\
\mathbf{Z} / 2 & =\left\langle\eta \beta^{n} y_{5}\right\rangle & 6 \\
\mathbf{Z} / 2^{4 n+6} \oplus \mathbf{Z} / 2^{2 n} & =\left\langle x_{8 n+7}\right\rangle \oplus\left\langle\bar{x}_{8 n+7}\right\rangle \\
\mathbf{Z} / 2 & =\left\langle\eta x_{8 n+7}\right\rangle & 7 \\
\mathbf{Z} / 2 \oplus \mathbf{Z} / 2 & =\left\langle\eta^{2} x_{8 n+7}\right\rangle \oplus\left\langle\beta^{n} y_{9}\right\rangle \\
\mathbf{Z} / 2 & =\left\langle\eta \beta^{n} y_{9}\right\rangle & 9 \\
& & 10
\end{array}\right.
$$

with relations $\eta^{2} \beta^{n} y_{5}=2^{4 n+5} x_{8 n+7}, \eta^{2} \beta^{n} y_{9} \equiv 2^{2 n+1} \bar{x}_{8 n+11} \bmod \left\langle 2^{4 n+6}\right\rangle$, and $\bar{x}_{n} \equiv \beta x_{n-8}-16 x_{n} \bmod \left\langle 32, \beta^{2}\right\rangle$ for all $n \geq 0$.

Remark. Note that the elements $\eta x_{n}$ and $\eta^{2} x_{n}$ are annihilated by $\beta$. Thus, the map $k o_{*} B S l_{2}(3) \rightarrow K O_{*} B S l_{2}(3)$ has a nontrivial kernel, unlike $k o_{*} B \mathbf{Z} / 2 \rightarrow K O_{*} B \mathbf{Z} / 2$ or $k o_{*} \Sigma^{-1} B S^{3} / B N \rightarrow K O_{*} \Sigma^{-1} B S^{3} / B N$.

Proof. The isomorphism of Corollary 3 ,

$$
K O_{*} B S l_{2}(3) \cong K O_{*} \mathbf{R} \mathbf{P}^{\infty} \oplus K S p_{*} \mathbf{R} \mathbf{P}^{\infty},
$$

implies that $K O_{4 n}=0, K O_{4 n+1} \cong K O_{4 n+2} \cong \mathbf{Z} / 2$, and that $\eta^{2}$ times $K O_{4 n+1}$ is nonzero.

We show first that this implies that $b^{n} y_{5}$ and $h_{1} b^{n} y_{5}$ survive to nonzero elements of $E_{\infty}$ for all $n \geq 0$. Clearly, their filtration is too high to support nontrivial differentials. On the other hand, if $b^{n} y_{5}$ is the target of a differential, then the same sort of infinite regress as in Theorem 4 leads to a contradiction. Therefore, $b^{n} y_{5}$ maps to the nonzero class in $\mathrm{KO}_{8 n+5} B S l_{2}(3)$, and hence, $\eta^{2}$ times it is nonzero. It follows that $h_{1} b^{n} y_{5}$ is also nonzero in $E_{\infty}$.

Now, all other classes in dimensions $8 n+4,8 n+5$, and $8 n+6$ must vanish upon inverting $b$. We show that this implies $d_{4}\left(y_{8 n+5}\right)=h_{1} b x_{8 n-5}$ by induction. Suppose $n=1$. If $d_{4}\left(y_{13}\right) \neq h_{1} b x_{3}$ then, for some $n$, we must have $b^{n} y_{13}$ the target of a differential. Say $d_{r}\left(h_{1} b^{n-i} y_{8 i+13}\right)=b^{n} y_{13}$. Since $b^{n} y_{13}$ is not an $h_{1}$ multiple, $h_{1} b^{n-i} y_{8 i+13}$ must be indecomposable under multiplication by $h_{1}$ in $E_{r}$. Hence $b^{n-i} y_{8 i+13}$ supports an earlier differential than $d_{r}$, and we are led to a contradiction as before. The induction now proceeds as in Theorem 4: $d_{4}\left(y_{8 n+5}\right)=h_{1} b x_{8 n-5}$ is the only possible nonzero differential on $y_{8 n+5}$ by the inductive hypothesis. The alternative possibility, that $b^{k} y_{8 n+5}$ is the target of a differential for some $k$, leads to a contradiction.

Now, the remaining survivors, $b^{n} y_{9}$ and $h_{1} b^{n} y_{9}$, and the remaining differentials $d_{4}\left(y_{8 n+1}\right)=h_{1} b x_{8 n-9}$, follow by exactly the same arguments.

In the resulting $E_{5}$-term, the indecomposables which might support differentials lie in dimensions $4 n-1$. The only remaining elements in dimensions $4 n-2$ must be nonzero in $E_{\infty}$. Hence, $E_{5}=E_{\infty}$.

The hidden $\eta$-extensions are propagated by multiplication by $\beta$ once $\eta^{2} y_{5}$ and $\eta^{2} y_{9}$ are determined. The first, $\eta^{2} y_{5}$, is inherited from the Moore space $M^{4}(8) \rightarrow$ $B l_{2}(3)$. The second, $\eta^{2} y_{9}$, is detected by considering

$$
B l_{2}(3) \leftarrow B^{8} \rightarrow B^{8} / B^{4}=M^{8}(8)
$$

where $B^{n}$ is the $n$-skeleton of $B S l_{2}(3)$. (See Figure 4.) 


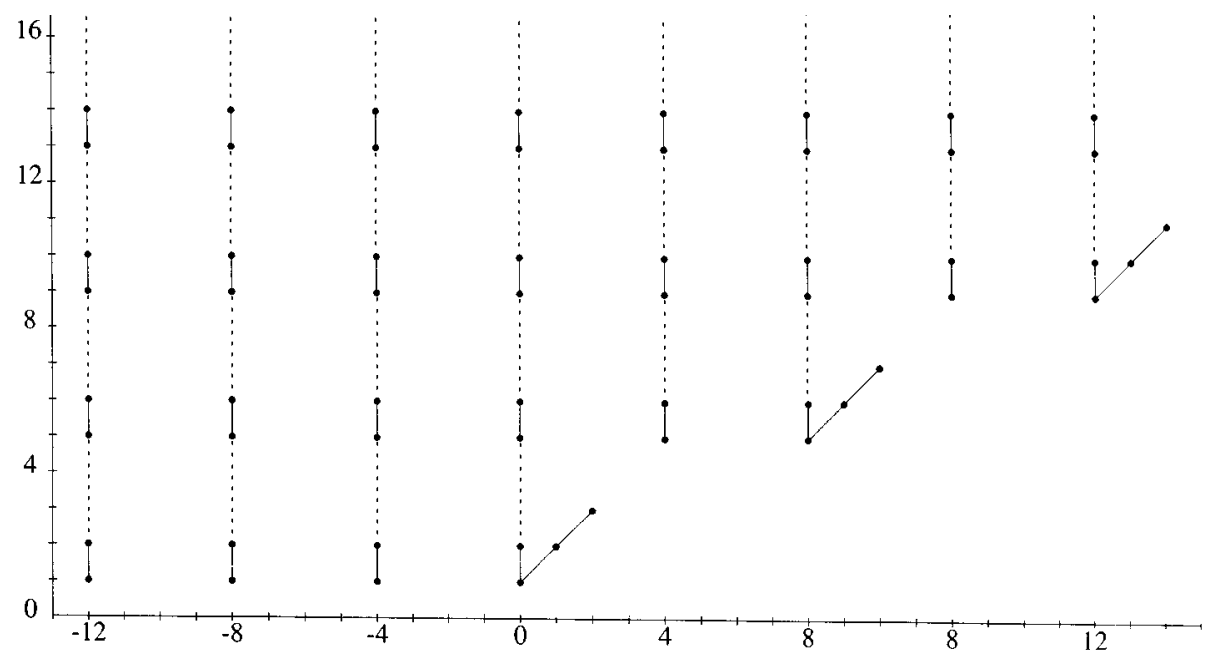

Figure 5. $E_{\infty}=E^{0}\left(k o^{-*} \Sigma^{-1} B S^{3} / B N\right)$

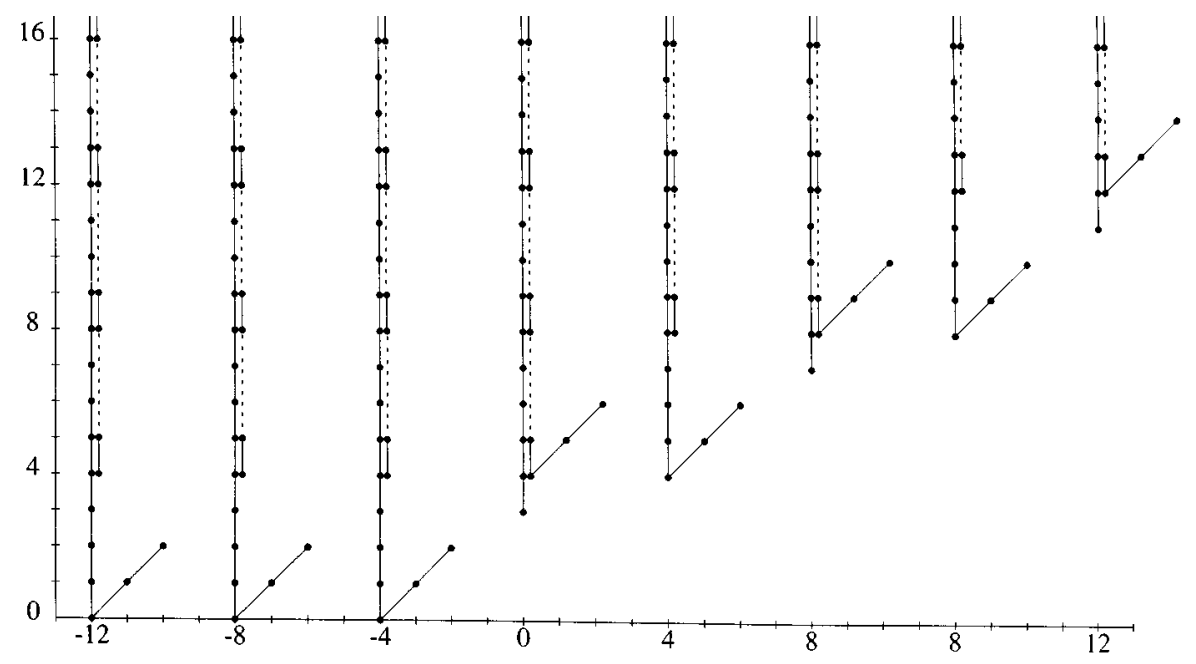

Figure $6 . E_{\infty}=E^{0}\left(k o^{-*} B S l_{2}(3)\right)$

It remains to determine the extensions in dimensions $4 n-1$. Multiplication by the Bott element is a monomorphism in dimensions $4 n-1$ in $E_{\infty}$, and hence in $k o_{*}$. Since $K O_{4 n-1} B S l_{2}(3)=\mathbf{Z} / 2^{\infty} \oplus \mathbf{Z} / 2^{\infty}, k o_{4 n-1} B S l_{2}(3)$ has rank $\leq 2$.

The $E_{\infty}$-term shows that $2^{4 n+3} \mathrm{ko}_{8 n+3}=0$ and $2^{4 n+6} \mathrm{ko}_{8 n+7}=0$. An element detected in filtration 0 will generate a subgroup of this order, which is therefore a direct summand. Dividing by this subgroup must yield a cyclic group, forcing the extensions. 


\section{The ko-COHOMOlOGy of $B G$}

The preceding calculations of ko-homology are easily dualized to obtain the following.

Theorem 6. There are classes $z_{n} \in k o^{4 n} \Sigma^{-1} B S^{3} / B N, n \geq 0$, which generate $k o^{*} \Sigma^{-1} B S^{3} / B N$ as a ko*-module, with relations $\eta z_{n}=0$ if $n \neq 0$, and $\beta z_{n}=$ $\omega z_{n-1}=4 z_{n-2}$. Thus

$$
k o^{i} \Sigma^{-1} B S^{3} / B N= \begin{cases}\widehat{\mathbf{Z}}_{2}, & i \equiv 0 \bmod 4, \\ \mathbf{Z} / 2, & i \equiv-1,-2 \bmod 4, i<0, \\ 0, & \text { otherwise. }\end{cases}
$$

Theorem 7. There are classes $z_{n} \in k o^{4 n} B S l_{2}(3), n>0$, which generate $k o^{*} B S l_{2}(3)$ as a $k o_{*}$-module, with relations $\eta \beta z_{n}=0$ if $n>2$, and $\omega z_{n+1} \equiv$ $8 z_{n} \bmod (8, \beta)$. Further,

$$
k o^{i} \operatorname{BSl}_{2}(3)= \begin{cases}\widehat{\mathbf{Z}}_{2} \oplus \widehat{\mathbf{Z}}_{2}, & i \equiv 0 \bmod 4, \\ \mathbf{Z} / 2, & i \equiv-1,-2 \bmod 4, \\ 0, & \text { otherwise. }\end{cases}
$$

Note: We will see that $z_{1}$ and $z_{2}$ each generate a copy of $k o_{*}$, while the other generators generate a copy of $k o_{*} /(\eta \beta)$.

We will prove these two results in tandem as follows. We can filter $B=B S l_{2}(3)$ and $X=\Sigma^{-1} B S^{3} / B N$ so that

$$
\begin{gathered}
B^{0}=X^{0}=*, \\
B^{n} / B^{n-1}=M^{4 n}(8), \\
X^{n} / X^{n-1}=M^{4 n-2}(2) .
\end{gathered}
$$

For convenience of indexing when we compute the coefficients of the Tate theory, we will henceforth index cohomology by the negative of the usual index. To calculate $k o^{-*} B S l_{2}(3)$ and $k o^{-*} \Sigma^{-1} B S^{3} / B N$ we use duality:

$$
\begin{aligned}
k o^{-q} B & =k o^{-q} \lim _{\rightarrow} B^{n} \\
& =\lim _{\leftarrow} k o^{-q} B^{n} \\
& =\lim _{\leftarrow} k o_{q} D B^{n}
\end{aligned}
$$

and similarly for $X=\Sigma^{-1} B S^{3} / B N$. It will emerge from the calculation of the inverse systems, that they are Mittag-Leffler and hence there are no $\lim ^{1}$-terms to consider. The finiteness of $D X^{n}$ and $D B^{n}$ implies the convergence of the inverse limit of the Adams spectral sequences.

The filtration of $D X^{n}$ induced by that of $X^{n}$ has filtration quotients

$$
D X^{-n} / M^{4 n+3}(2)=D X^{-n+1}
$$

for $n<0$. Thus, the inverse limit Adams spectral sequence for $k o^{-*} X$ has

$$
E_{2}=\bigoplus_{n=-\infty}^{-1} E_{2}\left(M^{4 n+3}(2)\right)
$$

Let $x_{n}$ and $y_{n}$ generate the $n^{\text {th }}$ summand here, as in $\S 3$, so that $\left|x_{n}\right|=(4 n+2,0)$ and $\left|y_{n}\right|=(4 n+4,1)$ for each $n<0$. The geometric boundary map for this filtration of $D X^{n}$, namely, the map

$$
M^{-4 n-1}(2) \longrightarrow D X^{n} \longrightarrow M^{-4 n-4}(2)
$$


is dual to the geometric boundary map

$$
M^{4 n+2}(2) \longleftarrow X^{n} \longleftarrow M^{4 n+5}(2) .
$$

Since the latter map induces the differential $d_{2}\left(x_{n+1}\right)=h_{1} y_{n}$, we see that the diagram

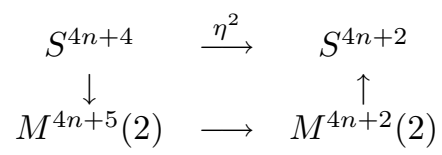

must commute after smashing with ko. Hence the dual diagram

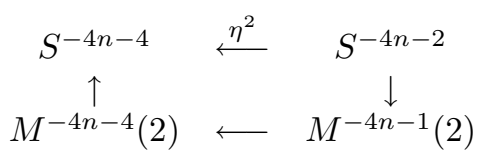

must also. This implies that the geometric boundary map for $D X^{n}$ also induces the differential

$$
d_{2}\left(x_{n+1}\right)=h_{1} y_{n}
$$

There are no further differentials possible. The resulting $E_{\infty}$-term is shown in Figure 5. Note that no elements of filtration zero survive to $E_{\infty}$.

The inverse limit Adams spectral sequence for $k o^{-*} B$ has

$$
E_{2}=\bigoplus_{n=-\infty}^{-1} E_{2}\left(S^{4 n}\right) \oplus E_{2}\left(S^{4 n+1}\right)
$$

with the first differential again implied by the Bockstein, leaving an $E_{4}$-term generated by $x_{4 n}$ and $y_{4 n+2},-\infty<n<0$, with $\left|x_{4 n}\right|=(4 n, 0)$ and $\left|y_{4 n+2}\right|=(4 n+2,1)$, and with the relation $w x_{4 n}=h_{0}^{3} x_{4 n+4}$. Applying duality as we did for $X$, we conclude that

$$
d_{4}\left(y_{4 n+2}\right)=h_{1} b x_{4 n-8}
$$

and that there are no further differentials possible. The extensions are resolved in the same way as they were for $k o_{*} B S l_{2}(3)$. The resulting $E_{\infty}$-term is shown in Figure 6.

Additively, $k o^{-*} B=\bigoplus \Sigma^{4 n} A$ summed over all integers $n$, where $A$ is the graded module $\hat{Z}_{2} \oplus \hat{Z}_{2}, Z / 2, Z / 2,0$ with generators $x, y, \eta x$, and $\eta^{2} x$, in degrees $0,0,1$, and 2 respectively. The action of the Bott generator $\beta \in k o^{-8}$ is more interesting. In negative degrees, i.e. $k o^{n} B$ with $n<0$, multiplication by $\beta$ is an isomorphism, as suggested by the right hand side of Figure 6 . In positive degrees however, multiplication by $\beta$ annihilates the $\eta$ multiples and introduces complicated relations among the torsion-free summands, as can be deduced from the hidden extensions in Figure 6. If we let $x_{n}$ be the filtration 0 generator of $\Sigma^{4 n} A$ in degree $4 n$, then

$$
4 \beta x_{n} \equiv 64 x_{n+2} \bmod \left\langle 128, \beta^{2}\right\rangle .
$$

We shall see in the next section that the norm sequence relating $k o_{*}, k o^{-*}$ and the Tate theory $t(k o)_{*}$ works to insert $k o_{*} B G$ and the $k o$-homology of a mod 8 Moore space coming from the disjoint basepoints in $k o_{q} B G_{+}$and $k o^{-q} B G_{+}$, into this picture in such a way that it becomes perfectly uniform. 


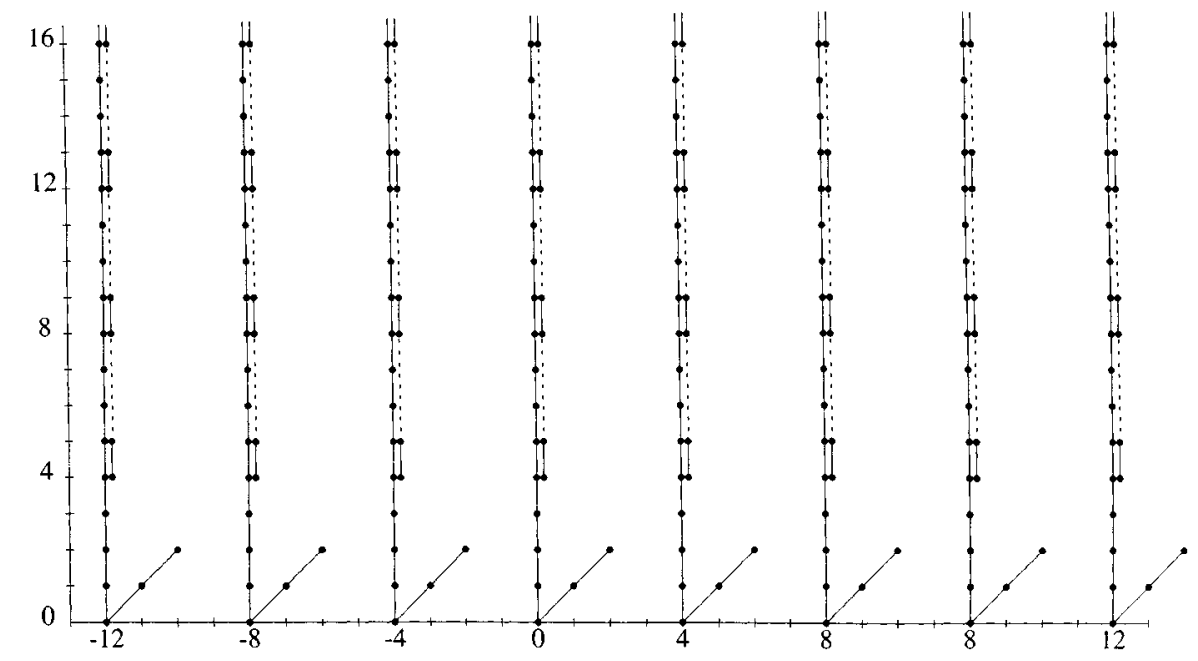

FiguRE 7. $E_{\infty}=E^{0}\left(t(k o)_{*}^{S l_{2}(3)}\right)$

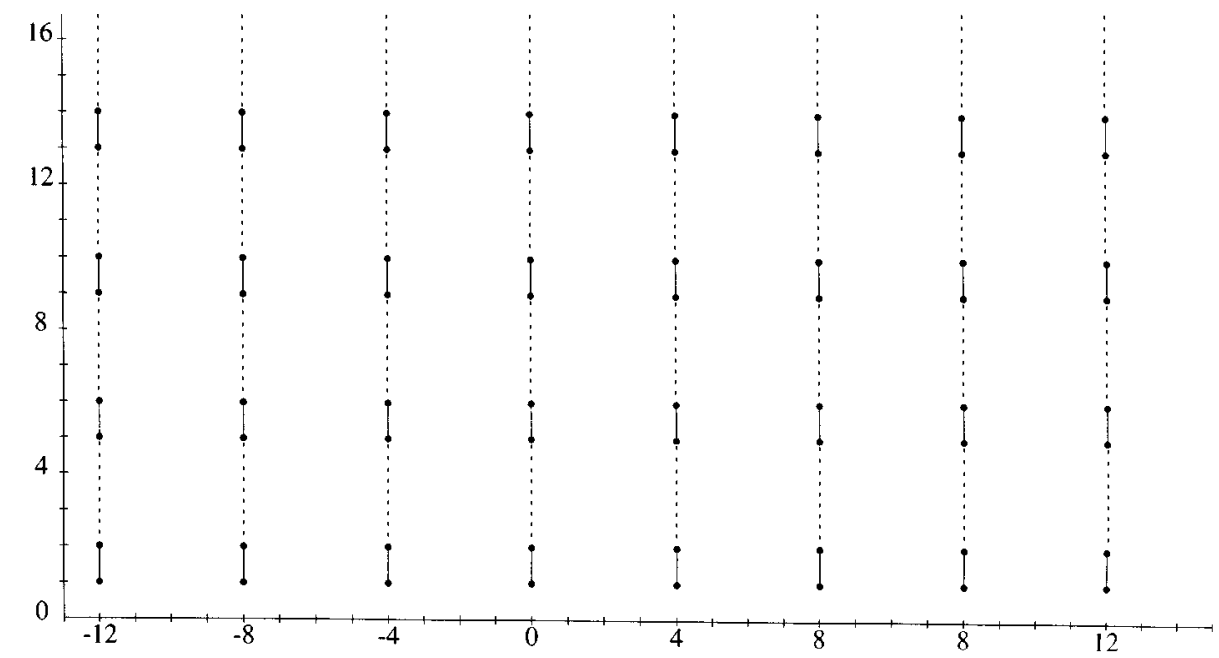

Figure 8. $E_{\infty}$ for the part of $E^{0}\left(t(k o)_{*}^{Q_{8}}\right)$ coming 'from $X$ '

\section{Coefficients of the Tate theory $t(k o)^{G}$}

Greenlees and May [9] have recently given a definition of a spectrum $t(k)$, which they call the Tate spectrum of $k$, for any $G$-spectrum $k$. Their definition agrees with previous, ad hoc, constructions of candidates for general 'Tate theories' and enjoys many good properties. Since generalized Tate theories are not yet widely known, we will discuss a few of these properties, then explain the conjecture which we disprove and the context which made it a reasonable conjecture. Finally, we suggest a minimal modification of the conjecture consistent with the results here. For a comprehensive treatment of this material, see [9]. Note that the construction 
takes place in the category of $G$-spectra, though some, but not all, of its good properties can be seen on the non-equivariant level by taking $G$-fixed points.

When $G$ acts freely on the unit sphere of a representation $V$, there is a particularly simple description of the $G$-fixed points $t(k)^{G}$,

$$
t(k)^{G}=\lim _{\leftarrow} B G^{\operatorname{Ad}(G)-n V} \wedge \Sigma k,
$$

where $A d(G)$ is the adjoint representation of $G$ on the tangent space at the identity and $B G^{\alpha}$ is the Thom spectrum of a virtual representation $\alpha$. When $G$ is $Z / 2, Z / p$ for an odd prime $p$, or $S^{1}$ this becomes

$$
\begin{gathered}
t(k)^{Z / 2}=\lim _{\leftarrow} \mathbb{R} P_{-n}^{\infty} \wedge \Sigma k, \\
t(k)^{Z / p}=\lim _{\leftarrow} \mathbb{L}_{-n}^{\infty} \wedge \Sigma k, \\
t(k)^{S^{1}}=\lim _{\leftarrow} \mathbb{C} P_{-n}^{\infty} \wedge \Sigma^{2} k
\end{gathered}
$$

where the extra suspension in the last case comes from $\operatorname{Ad}\left(S^{1}\right)=\mathbb{R}$. (Here $\mathbb{R} P_{-n}^{\infty}$, $\mathbb{L}_{-n}^{\infty}$ and $\mathbb{C} P_{-n}^{\infty}$ are the usual stunted real projective space, lens space and complex projective space, respectively.) Thus, Tate theories provide a vast generalization of Mahowald's remarkable root invariant inverse system and spectral sequence, which led to Lin's theorem and the proof of the Segal conjecture. We shall exploit this isomorphism for $G=Q_{8}$. Let $Q P_{-n}^{\infty}$ be the Thom space of the virtual representation $-n \mathbb{H}$, where $\mathbb{H}$ is the quaternions with the usual $Q_{8}$ action. Then

$$
t(k)^{Q_{8}}=\lim _{\leftarrow} Q P_{-n}^{\infty} \wedge \Sigma k .
$$

James periodicity applied to the finite stunted projective spaces $Q P_{m}^{n}$ will allow us to determine $t(k)_{*}^{Q_{8}}$ immediately from the calculations already done.

Perhaps the most remarkable property of Greenlees and May's construction of Tate spectra is the fact that $t(k)$ is a ring spectrum when $k$ is, and that the natural map $k \rightarrow t(k)$ is a ring map. In particular, this shows that the root invariant spectral sequence, properly graded, is multiplicative, a fact which is not apparent from ad hoc constructions of Tate spectra.

The properties which one would expect of a Tate theory are, first, that it vanishes on free $G$-spaces (or spectra), second, that it represents classical Swan-Tate homology if $k$ is ordinary homology and $G$ is finite, and, third, that there is a long exact norm sequence

$$
\cdots \rightarrow t(k)_{n+1}^{G} \rightarrow k_{n} B G_{+} \rightarrow k^{-n} B G_{+} \rightarrow t(k)_{n}^{G} \rightarrow \cdots
$$

in which the middle map is a generalized norm map which is simply multiplication by the Euler characteristic of $G$ on the components coming from the disjoint basepoint of $B G_{+}$.

Roughly speaking, for K-theory, the norm sequence exhibits $k$-cohomology as a lattice in the Tate theory $t(k)$, with quotient the suspension of the $k$-homology. For periodic K-theory, and 2-groups, this is a sum of copies of the sequence $\widehat{\mathbb{Z}}_{2} \rightarrow$ $\mathbb{Q}_{2} \rightarrow \mathbb{Q}_{2} / \widehat{\mathbb{Z}}_{2}$, in which the connective K-theory is imbedded as a sum of copies of $\widehat{\mathbb{Z}}_{2} \rightarrow \widehat{\mathbb{Z}}_{2} \rightarrow \mathbb{Z} / 2^{n}$ for various $n$. More precisely, Greenlees and May have shown that $t(K)$ and $t(K O)$ are rational [9]. (In general, Greenlees and Sadofsky have shown that if $k$ is a $v_{n}$-spectrum, the $t(k)^{G}$ is a $v_{n-1}$-spectrum [8].) The norm sequence for periodic K-theory then takes the form shown in the following table, in which the maps of the norm sequence are the obvious natural maps. Let $R O$, 
$R U$, and $R S p$ be the real and complex representation rings and the quaternionic representation module. Let $J O$ and $J S p$ be the kernels of the augmentation, and let $\widehat{()}$ denote $J O$-adic completion.

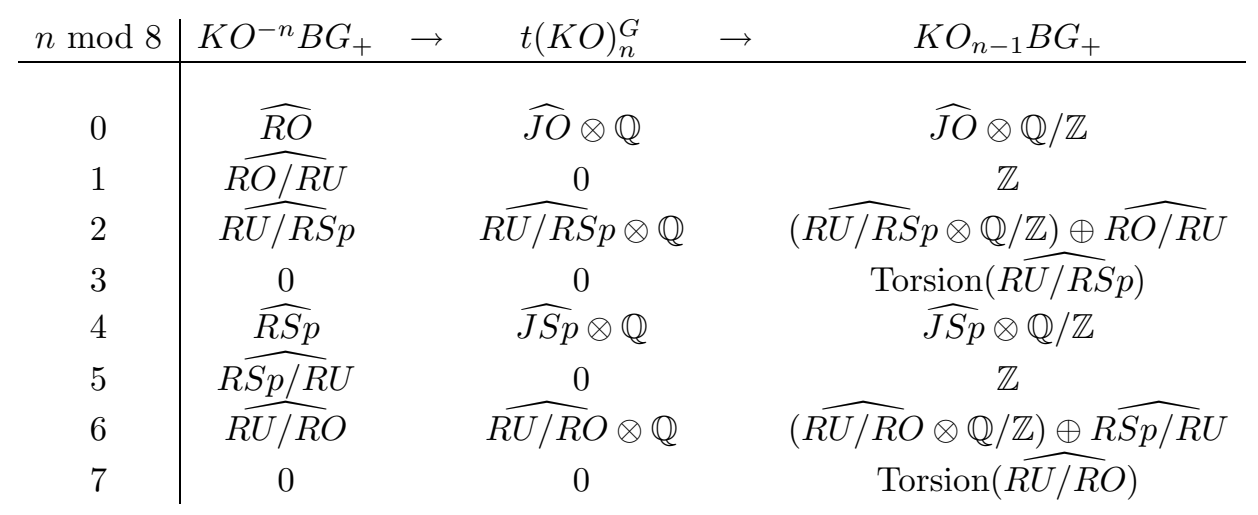

Since rational spectra split into a wedge of Eilenberg-Mac Lane spectra, it follows that $t(K O)^{G}$ is the wedge of rational Eilenberg-Mac Lane spectra determined by the right hand column above. Greenlees and May conjectured that the following analog of this fact holds for $t(k o)^{G}$.

Conjecture 8 ([9], Conj. 13.4). With the wedge taken over all integers $i$,

$$
\begin{aligned}
t(k o) \simeq & \vee K(\widehat{J O}, 8 i) \vee K((R \widehat{U / R S p}) / \text { torsion, } 8 i+2), \\
& \vee K(\widehat{J S p}, 8 i+4) \vee K((\widehat{R U / R O}) / \text { torsion, } 8 i+6) .
\end{aligned}
$$

The conjecture holds for $G$ of prime order by results of Davis and Mahowald [4]. However, the conjecture fails for $G=Q_{8}$ or $S l_{2}(3)$ for the following reason. It would imply that $t(k o)_{*}^{G} \longrightarrow t(K O)_{*}^{G}$ is a monomorphism. However, the Bott element acts invertibly on $t(K O)_{*}^{G}$, but annihilates some elements of $t(k o)_{*}^{G}$, so this is impossible. Also, if $t(k o)^{G}$ is to split into Eilenberg-Mac Lane spectra, then $\eta$ will act trivially on $t(k o)_{*}^{G}$. Our calculation shows that there are nontrivial $\eta$ multiples in $t(k o)_{*}^{G}$. In fact, these are the elements which are annihilated by the Bott element. Thus, eliminating the elements annihilated by the Bott element leads to the following minimal modification to the conjecture consistent with our calculations here.

Conjecture 9. There exist a spectrum $t(k o) /(\operatorname{ker} \beta)$ and maps

$$
t(k o) \longrightarrow t(k o) /(\operatorname{ker} \beta) \longrightarrow t(K O)
$$

factoring the natural map. Further, $t(k o) /(\operatorname{ker} \beta)$ splits into Eilenberg-Mac Lane spectra as in Conjecture 8.

Our calculation which disproves Conjecture 8 and suggests our conjecture is as follows.

Theorem 10. If $G=Q_{8}$ or $S l_{2}(3)$ then, 2-locally, the coefficients of the Tate $G$-spectrum of ko are

$$
t(k o)_{*}^{Q_{8}}=t(k o)_{*}^{S l_{2}(3)} \oplus \bigoplus_{n=-\infty}^{\infty} \Sigma^{4 n}\left(\hat{Z}_{2} \oplus \hat{Z}_{2}\right)
$$


and

$$
t(k o)_{*}^{S l_{2}(3)}=\bigoplus_{n=-\infty}^{\infty} \Sigma^{4 n} A
$$

where $A$ is the graded module $\left(\hat{Z}_{2} \oplus \hat{Z}_{2}, Z / 2, Z / 2,0\right)$, with generators $x, y$, $\eta x$, and $\eta^{2} x$.

Proof. We may assemble the Adams spectral sequences converging to

$$
\begin{aligned}
& k o^{-*} B G_{+}=\pi_{*} F\left(B G_{+}, k o\right), \text { and } \\
& k o_{*} \Sigma B G_{+}=\pi_{*} k o \wedge \Sigma B G_{+}
\end{aligned}
$$

in standard fashion to get a spectral sequence converging to $\pi_{*} t(k o)^{G}$ with differentials derived from the differentials in the two halves and from the boundary map

$$
k o \wedge B G_{+} \stackrel{\tau}{\longrightarrow} F\left(B G_{+}, k o\right) .
$$

However, the differentials implied by $\tau$ are difficult to determine in general. When $G=Q_{8}$, periodicity implies them as follows. We use the description of the Tate spectrum as

$$
t(k o)^{Q_{8}}=Q P_{-\infty}^{\infty} \wedge \Sigma k o=\lim _{\leftarrow} Q P_{-n}^{\infty} \wedge \Sigma k o
$$

as in [9, Thm 16.1], where $Q P_{-n}^{\infty}$ is the Thom complex of $-n$ times the quaternionic line bundle over $B Q_{8}$. The differentials in the resulting Adams-AtiyahHirzebruch spectral sequence are then periodic by James periodicity applied to the finite stunted quaternionic projective spaces. As a consequence, the differentials which hold in the Adams spectral sequence for $k o^{-n} B G, G=Q_{8}$ or $B S l_{2}(3)$, for $n<0$, hold for all $n$ in the spectral sequence for $t(k o)_{*}^{G}$. A similar periodicity applies to the extension questions. The resulting $E_{\infty}$-terms for $t(k o)_{*}^{B S l_{2}(3)}$ and each of the two other identical pieces of $t(k o)_{*}^{Q_{8}}$ are displayed in Figures 7 and 8 . QED

\section{REFERENCES}

1. J. F. Adams, Stable Homotopy and Generalised Homology, Chicago Lectures in Mathematics, University of Chicago Press, Chicago, 1974. MR 53:6534

2. D. W. Anderson, E. H. Brown, Jr., and F. P. Peterson, Pin cobordism and related topics, Comment. Math. Helv. 44 (1969), 462-468. MR 41:6226

3. Dilip Bayen, The connective real K-homology of finite groups, Thesis, Wayne State University, 1994.

4. D.M. Davis and Mark Mahowald, The spectrum $(P \wedge b o)_{-\infty}$, Math. Proc. Camb. Phil. Soc. 96 (1984), 85-93. MR 85j:55018

5. Boris Botvinnik and Peter Gilkey, The eta invariant and metrics of positive scalar curvature, Math. Annalen (to appear).

6. Boris Botvinnik and Peter Gilkey, Metrics of positive scalar curvature on spherical space forms, Can. J. Math (to appear).

7. Boris Botvinnik, Peter Gilkey and Stephan Stolz, The Gromov-Lawson-Rosenberg conjecture for manifolds with a spherical space form fundamental group, Preprint of IHES, November, 1995.

8. J. P. C. Greenlees and Hal Sadofsky, The Tate spectrum of $v_{n}$-periodic complex oriented theories, Math. Zeit. (to appear).

9. J. P. C. Greenlees and J. P. May, Generalized Tate cohomology, Memoirs AMS $\mathbf{5 4 3}$ (1995). CMP 95:07

10. J. Martino and S. B. Priddy, Classification of BG for groups with dihedral or quaternion Sylow 2-subgroup, J. Pure. Appl. Alg. 73 (1991), 13-21. MR 92f:55022

11. J. P. May and R. J. Milgram, The Bockstein and the Adams spectral sequences, Proc. AMS 83(1) (1981), 128-130. MR 82i:55019 
12. M. Mahowald and R. J. Milgram, Operations which detect $S q^{4}$ in connective $K$-theory and their applications, Quart. J. Math. Oxford (2), 27 (1976), 415-432. MR 55:6429

13. S. B. Priddy and Z. Fiedorowicz, Homology of Classical Groups Over Finite Fields and Their Associated Infinite Loop Spaces, Lecture Notes in Math. no 674, Springer-Verlag, 1978. MR 80g:55018

14. Stephan Stolz, Splitting MSpin-module spectra, Topology 33 (1994). MR 94m:55005

15. S. Stolz and J. Rosenberg, A stable version of the Gromov-Lawson conjecture, The Čech Centennial, Contemp. Math., vol. 181, Amer. Math. Soc., Providence, RI, 1995. CMP 95:09

16. R. E. Stong, Determination of $H^{*}(B O(k, \ldots, \infty) ; Z / 2)$ and $H^{*}(B U(k, \ldots, \infty)$, Trans. AMS 107 (1963), 526-544. MR 27:1944

Department of Mathematics, Wayne State University, Detroit, Michigan 48202

E-mail address: dbayen@math.wayne.edu

E-mail address: rrb@math.wayne.edu 